\title{
Aplicações do cálculo numérico para resolução de equações transcendentes na mecânica quântica
}

Applications of the numerical calculation for a resolution of transcendent equations in quantum mechanics

\author{
M.S. Maior de Sousa*1], F.A do Nascimento ${ }^{1}$ \\ ${ }^{1}$ Universidade Federal de Roraima, Centro de Educação, Colégio de Aplicação, B. Vista, RR, Brasil.
}

\begin{abstract}
Recebido em 18 de junho de 2019. Revisado em 01 de dezembro de 2019. Aceito em 06 de agosto de 2020.
Muitos estudantes de física e de certos cursos de engenharia se deparam com a disciplina de cálculo numérico, desenvolvendo-a de maneira puramente abstrata. Desta forma, este trabalho visa o uso do calculo numérico como ferramenta para soluções de problemas envolvendo a mecânica quântica com o intuito de demonstrar na prática a sua aplicação na obtenção e análise de resultados em dois problemas específicos: $(i)$ O poço de potencial duplo tipo $\delta(x)$ simétrico. (ii) E o poço quadrado potencial infinito com uma barreira de potencial centrada, tipo $\delta(x)$. Para estes dois casos mostramos que as soluções possíveis de energias permitidas podem ser obtidas pelo cálculo numérico, especificamente pelo método da bisseção. Mostramos também que a paridade da função de onda interfere diretamente nos estados permitidos e nos efeitos sobre a partícula submetida a estes dois potenciais.
\end{abstract}

Palavras-chave: Cálculo numérico, Equação de Schrodinger, Potencial $\delta$, Análise numérica.

\begin{abstract}
Many students of physics and of certain courses of engineering are faced with the discipline of numerical calculation, developing it in a purely abstract way. In this way, this work aims at the use of numerical calculus as a tool to solve problems involving quantum mechanics in order to demonstrate in practice its application in obtaining and analyzing results in two specific problems: $(i)$ The double well symmetric potential $\delta(x)$. (ii) And the square well infinite potential with a centered potential barrier, type $\delta(x)$. For these two cases we show that the possible solutions of allowed energies can be obtained by the numerical calculation, specifically by the method of the bisection. We also show that the parity of the wave function interferes directly in the allowed states and the effects on the particle subjected to these two potentials.
\end{abstract}

Keywords: General Relativity, Field Theory, Gravitation, Abelian Votex.

\section{Introdução}

Na mecânica quântica, a equação de Schrödinger é uma equação diferencial parcial que descreve como o estado quântico de um sistema físico evolui no tempo. Esta por sua vez, foi formulada por volta de 1925, e publicado em 1926, pelo físico austríaco Erwin Schrödinger [1]. A formulação da descrição quântica de uma função de onda é um postulado fundamental da mecânica quântica. Na interpretação padrão da mecânica quântica, a função de onda é a descrição mais completa que pode ser dada a um sistema físico. As soluções para a equação de Schrödinger descrevem não só sistemas moleculares, atômicas e subatômicas, mas também certos sistemas macroscópicos.

A análise da equação de Schrödinger com um potenciais específicos envolvendo a função $\delta(x)$ de Dirac estão fortemente presentes nas páginas de muitos livros [1]- [7]. Os possíveis estados ligados podem ser obtidos por meio de uma solução direta da equação de Schrödinger com apoio da descontinuidade da derivada primeira da autofunção, mais a continuidade da autofunção e seu comportamento assintótico. Na referência 8 tal problema foi examinado

*Endereço de correspondência: mikael.souto@ufrr.br com o método da transformada de Laplace, onde os autores mostraram que solução do problema de estados ligados pode ser obtida sem que haja alguma informação da descontinuidade da derivada primeira da autofunção. Na referência 9] O problema de um simples potencial delta resume-se à resolução de uma equação algébrica de primeiro grau para a transformada de Fourier da autofunção e o problema para mais que uma função delta também revela-se uma questão simples.

Entretanto, em certo ponto, alguns comportamentos quânticos de um determinando sistema não podem ser descritos de maneira analítica em sua totalidade. Desta forma, é muito comum nesta área utilizar-se de métodos computacionais e/ou métodos numéricos para determinar soluções em determinados problemas na mecânica quântica 10. Assim, este trabalho trás uma abordagem numérica para tais problemas quânticos já conhecidos analiticamente. Vamos determinar as autoenergias dos estados ligados para o problema do poço de potencial duplo simétrico do tipo $\delta(x)$, onde mostramos que as soluções pares admitem pelo menos dois estados ligados, enquanto que as soluções ímpares admitem apenas um estado ligado. E, em seguida, vamos abordar o problema 
do poço quadrado infinito de largura $2 a$, com uma função $\delta(x)$ centrada na origem.

Nos dois casos abordados, veremos que aparecerão equações denominadas de equações transcendentes [11]. Uma equação transcendente é uma equação que contém alguma função que não é redutível a uma fração entre polinômios, e cuja solução não pode ser expressa através de funções elementares. De modo geral, uma equação transcendente não possui uma solução exata expressa através de funções conhecidas, sendo necessário recorrer ao cálculo numérico para obter uma solução.

\section{A equação de Schrodinger}

Na mecânica quântica, a equação de Schrödinger é uma equação diferencial parcial que descreve como o estado quântico de um sistema físico muda com o tempo. Foi formulada no final de 1925, e publicado em 1926, pelo físico austríaco Erwin Schrödinger 1,7].

Seja $\Psi(x, t) \equiv \Psi$ a função de onda da partícula, escrevemos a equação de Schrödinger da seguinte forma:

$$
i \hbar \frac{\partial \Psi}{\partial t}=-\frac{\hbar^{2}}{2 m} \frac{\partial^{2} \Psi}{\partial x^{2}}+V \Psi .
$$

A partir da equação (1) conhecendo as condições iniciais, isto é, conhecendo $\Psi(x, 0)$ podemos determinar a evolução temporal da partícula.

Por si só a função de onda nada mais é do que uma representação abstrata do estado do sistema. Ela só tem significância no contexto da mecânica quântica. Além disso, por ser uma quantidade complexa, ela não pode ser medida diretamente por um instrumento físico.

Neste sentido, em 1926, Max Born propôs uma interpretação probabilística pra a função de onda, ou seja:

$$
p(x, t)=|\Psi(x, t)|^{2},
$$

onde $p(x, t)$ é a probabilidade de encontrar a partícula na posição $x$ num certo instante $t$. Obviamente, em todo espaço a probabilidade de encontrarmos a partícula em um certo ponto num certo tempo deve ser tal que:

$$
\int d x|\Psi(x, t)|^{2}=1
$$

essa condição determina a normalização da função de onda.

Os estados estacionários são extremamente importantes na descrição quântica da natureza, não só porque representam estados de energia definida, mas também por formar um conjunto completo dos autoestados do hamiltoniano. Isto significa que qualquer estado pode ser representado como uma combinação linear de estados estacionários.

A determinação dos estados estacionários de um determinado hamiltoniano é feita normalmente resolvendo-se a equação de Schrödinger independente do tempd ${ }^{1}$ que

${ }^{1}$ Esta equação é obtida a partir de 1 , onde considera-se um potencial dependente apenas da posição $V(x)$, fazendo uma separação de variáveis, isto é, tomando $\Psi(x, t)=\psi(x) \phi(t)$ é dada na seguinte forma:

$$
H \psi(X)=-\frac{\hbar^{2}}{2 m} \frac{d^{2}}{d x^{2}} \psi(x)+V(x) \psi(x),
$$

cuja operação sobre a função de onda estacionária determina a energia $E$, isto é, $H \psi(x)=E \psi(x)$.

\section{Proposição do problema}

O intuito deste trabalho é desenvolver as aplicações da mecânica quântica no contexto do cálculo numérico, uma vez que, trabalhando com certas aplicações da mecânica quântica, um desenvolvimento analítico pode se tornar inviável, de contrapartida podemos utilizar a análise numérica, principalmente quando tratamos de aplicações que geram soluções de autoenergias bem definidas a partir de equações transcendentes.

Nos casos a seguir iremos tratar dos seguintes problemas propostas: $(i) \mathrm{O}$ potencial duplo tipo $\delta(x)$. E o (ii) O poço de potencial quadrado infinito com uma descontinuidade, $\delta(x)$, na origem.

\subsection{O potencial duplo tipo $\delta(x)$}

Vamos resolver a equação de Schrödinger definida pela equação (4), tomando o potencial da seguinte forma:

$$
V(x)=-\alpha[\delta(x-a)+\delta(x+a)]
$$

onde $\alpha$ é um parâmetro que tem dimensão de energia.

Este tipo de potencial é graficamente representado de acordo com a Figura 1, de acordo com 7 ].

Vamos estudar, neste caso, os estados ligados envolvendo este tipo de situação e resolver o problema das autoenergias permitidas através da análise numérica, para isto nos reservamos ao caso em que $E \leq V(x)$.

Utilizando a equação estacionária de Schrödinger, vamos analisar separadamente as regiões $(I),(I I)$ e $(I I I)$, para os três caso é visto que para $|x| \neq a$ temos:

$$
\frac{d^{2} \psi(x)}{d x^{2}}=k^{2} \psi(x)
$$

com

$$
k^{2}=-\frac{2 m E}{\hbar^{2}} .
$$

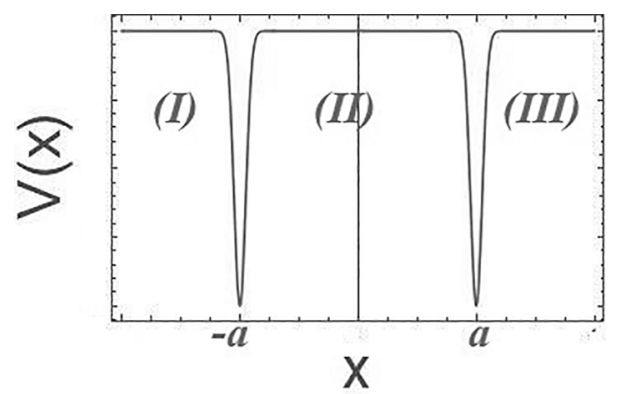

Figura 1: Representação do esquemática do potencial duplo delta de Dirac, nos pontos $\pm a$ o potencial possui uma descontinuidade. 
Antes de darmos continuidade com o problema de determinar os estados ligados e as autoenergias vamos lembra de uma propriedade geral das soluções da equação se Schrödinger, na qual refere-se a casos onde o potencial é uma função par, ou seja, $V(x)=V(-x)$. Neste caso, verifica-se que as autofunções $\psi(x)$ e $\psi(-x)$ satisfazem a equação estacionária de Schrödinger com a mesma autoenergia $E$. De fato, combinações lineares dessas funções também são soluções como a mesma autoenergia. Em particular temos:

- Se a função for uma combinação par:

$$
\psi^{(+)}(x)=\psi(x)+\psi(-x)
$$

- Se a função for uma combinação ímpar:

$$
\psi^{(-)}(x)=\psi(x)-\psi(-x)
$$

A equação (6) é uma equação diferencial ordinária com coeficientes constantes cuja solução geral é da forma

$$
\psi_{j}^{( \pm)}(x)=A_{j} e^{k x} \pm B_{j} e^{-k x}
$$

onde o índice $j$ representa a região específica, ou seja, para $j=1$ tratamos da região $(I)$ e assim por diante até a região $(I I I)$.

Tratando-se de estados ligados, isto é, cujas funções de onda sejam normalizáveis ${ }^{2}$. Analisando para $j=1$, ou seja, para a região $(I)$ temos que $B_{1}=0$ e assim,

$$
\psi_{1}(x)=A_{1} e^{k x}
$$

De modo análogo analisando a região $(I I I)$ temos que em $x \rightarrow+\infty$ para que a função de onda seja normalizável é necessário que $A_{3}=0$ assim temos:

$$
\psi_{3}(x)=B_{3} e^{-k x} .
$$

Para a região $(I I)$ a partícula está confinada no intervalo de $(-a, a)$ assim temos:

$$
\psi_{2}(x)=A_{2} e^{k x}+B_{2} e^{-k x} .
$$

Vamos considerar inicialmente que a função de onda $\psi(x)$ seja uma função par, assim temos que $\psi(-a)=$ $\psi(a), \operatorname{logo}$ temos para a região $(I)$ e região $(I I I)$ que $A_{1}=B_{3}=A$, assim,

$$
\begin{aligned}
& \psi_{1}^{(+)}(x)=A e^{k x} \\
& \psi_{3}^{(+)}(x)=A e^{-k x}
\end{aligned}
$$

Além disso, a função de onda deve ser contínua em todos os pontos, isto implica que em $x= \pm a$ temos:

$$
\begin{aligned}
\psi_{1}^{(+)}(-a) & =\psi_{2}^{(+)}(-a) \\
\psi_{3}^{(+)}(a) & =\psi_{2}^{(+)}(a)
\end{aligned}
$$

${ }^{2}$ Para que uma função de onde seja normalizável é necessário que no limite $x \rightarrow \pm \infty$ tenhamos $\psi(x) \rightarrow 0$. o que de fato só é verdadeiro se $A_{2}=B_{2}$. Assim, o conjunto solução é tal que

$$
\begin{aligned}
\psi_{1}^{(+)}(x) & =A e^{k x} \\
\psi_{2}^{(+)}(x) & =A_{2}\left(e^{k x}+e^{-k x}\right) \\
\psi_{3}^{(+)}(x) & =A e^{-k x} .
\end{aligned}
$$

Seguindo o procedimento análogo, considerando agora a função de onda como uma combinação linear ímpar, temos que o conjunto solução é tal que

$$
\begin{aligned}
& \psi_{1}^{(-)}(x)=-A e^{k x} \\
& \psi_{2}^{(-)}(x)=A_{2}\left(e^{k x}-e^{-k x}\right) \\
& \psi_{3}^{(-)}(x)=A e^{-k x} .
\end{aligned}
$$

Nosso problema agora consistirá em determinar as autoenergias dos estados ligados quando considerarmos soluções pares ou ímpares. Para isso vamos aplicar a condição de descontinuidade da derivada primeira da função de onda sobre o ponto onde o potencial é divergente [1,7], ou seja, em $x= \pm a$. Contudo, pela simetria do problema, basta desenvolvemos a equação para $x=a$ que por consequência encontraremos a solução para $x=-a$ e vice-versa.

Por conveniência, para não carregar notação, vamos tomar a $\psi^{(+)}(x) \equiv \psi(x)$ para as soluções cuja função de onda seja par, daí segue que, pela condição de descontinuidade da derivada da função de onda no ponto onde o potencial diverge temos:

$$
\lim _{\epsilon \rightarrow a}\left(\psi^{\prime}(\epsilon)_{\epsilon^{+}}-\psi^{\prime}(\epsilon)_{\epsilon^{-}}\right)=-\frac{2 m \alpha}{\hbar^{2}} \psi(a)
$$

onde $\epsilon^{ \pm}$denote a aproximação do do ponto $a$ pela direita e pela esquerda respectivamente.

Por outro lado, para $\psi(a)$, na equação 24 temos para o membro após a igualdade que:

$$
\psi(a)=\psi_{3}(a)=A e^{-k a}
$$

Já para o membro antes da igualdade temos para $\epsilon^{+} \rightarrow a$ que

$$
\psi^{\prime}(\epsilon)_{\epsilon^{+}}=\psi_{3}^{\prime}(a)=-k A e^{-k a},
$$

e por sua vez

$$
\psi^{\prime}(\epsilon)_{\epsilon^{-}}=\psi_{2}^{\prime}(a)=-k A_{2}\left(e^{-k a}-e^{k a}\right) .
$$

Substituindo as equações (25), 26) e (27) na equação (24) obtemos a relação entre as constantes $A_{2}$ e $A$ de tal forma que

$$
A_{2}=\frac{\frac{2 m \alpha}{\hbar^{2} k}-1}{e^{2 k a}-1} A
$$

Usando o resultado acima na condição de continuidade da função de onda, isto é, em

$$
\psi_{3}(a)=\psi_{2}(a)
$$


encontramos a seguinte equação:

$$
e^{-2 k a}=\frac{\hbar^{2} k}{m \alpha}-1
$$

E seguindo o mesmo procedimento para soluções ímpares temos:

$$
e^{-2 k a}=-\frac{\hbar^{2} k}{m \alpha}+1
$$

As equações 30 e 31 não possuem soluções analíticas, apenas soluções numéricas, estes tipos de equações são conhecidas como equações transcendentes e estão muito presentes em vários problemas teóricos na física. Resolvendo estas equações para a variável $k$ encontramos as autoenergias dos estados ligados para partículas quânticas submetidas a um potencial tipo $\delta$ de Dirac.

\subsection{O poço de potencial infinito com uma função $\delta(x)$ centrada na origem}

Nesta subseção vamos tratar do problema do poço quadrado de potencial infinito, isto é, o problema no qual o potencial $V(x) \rightarrow \infty$ para $x= \pm a$ e, $V(x)=0 \forall x \in$ $(-a, a)$, onde a distância de $-a$ até $a$ é a largura do poço. Sendo que, agora este potencial sofrerá uma descontinuidade na origem, isto é, $V(x)=\alpha \delta(x)$.

Assim como no caso do potencial duplo tipo função delta de Dirac, desenvolvido na subseção anterior, este potencial é simétrico, isto é, fazendo $x \rightarrow-x$ o potencial preserva a paridade. Logo, a solução geral pode ser dada por uma função par ou ímpar.

Sabe-se que para o poço de potencial quadrado infinito a solução geral [1 1 2 7] para a equação de onda Schrödinger é tal que:

$$
\psi(x)=A \sin (k x)+B \cos (k x) .
$$

Como o potencial sofre uma descontinuidade em $x=0$ podemos pensar no problema proposto como dois poços quadrados infinitos, onde uma vai de $-a$ até zero, e o outro de zero até $a$. Desta maneira, podemos dizer que (32) seria a solução para o poço contido no intervalo $(0, a]$. Para determinar a solução no intervalo entre $[-a, 0)$ basta tomar a substituição $x \rightarrow-x$ em 32 considerando a paridade da função de onda.

Para a função de onda com paridade negativa (função ímpar) temos $\psi(x)=-\psi(-x)$. Desta maneira, a partir de 32 temos a seguinte solução:

$$
\psi(x)=A \sin (k x)-B \cos (k x) \forall x \in[-a, 0) .
$$

Aplicando a continuidade da função de onda em $x=0$ ver-se que $B=0$. Daí o conjunto solução é tal que:

$$
\psi(x)=\left\{\begin{array}{r}
A \sin (k x) \forall x \in(0, a] \\
A \sin (k x) \forall x \in[-a, 0)
\end{array}\right.
$$

Aplicando a condição de nulidade da função de onda em $x= \pm a$ segue que:

$$
k a=\frac{n \pi}{2} \forall n=2,4,6,8 \ldots
$$

Considerando que as soluções dentro do poço são as soluções que devem satisfazer (2), onde agora consideramos:

$$
k^{2}=\frac{2 m E}{\hbar^{2}}
$$

temos que as autoenergias possíveis são dadas por:

$$
E_{n}=\frac{n^{2} \pi^{2} \hbar^{2}}{2 m(2 a)^{2}} \forall n=2,4,6,8 \ldots
$$

Ou seja, para soluções ímpares a partícula não sente o efeito da descontinuidade.

Vamos analisar agora a função de onda com paridade positiva (soluções com paridade par). Neste caso, tomando a equação (32), com a transformação $x \rightarrow-x$ temos que o conjunto solução é tal qual:

$$
\psi(x)=\left\{\begin{array}{r}
A \sin (k x)+B \cos (k x) \forall x \in(0, a] \\
-A \sin (k x)+B \cos (k x) \forall x \in[-a, 0)
\end{array}\right.
$$

Aplicando a continuidade da função de onda em $x=0$ para o conjunto solução (38), temos diretamente que $B=B$. Por sua vez, aplicando a descontinuidade da derivada da função de onda em $x=0$, dada pelo conjunto solução 38 , determinamos a relação entre as constantes $A$ e $B$, assim temos:

$$
B=\frac{k \hbar^{2}}{2 m a} A
$$

e podemos reescrever o conjunto solução como

$$
\psi(x)=\left\{\begin{array}{r}
A\left(\sin (k x)+\frac{k \hbar^{2}}{2 m a} \cos (k x)\right) \forall x \in(0, a] \\
-A\left(\sin (k x)+\frac{k \hbar^{2}}{2 m a} \cos (k x)\right) \forall x \in[-a, 0)
\end{array}\right.
$$

Como queremos soluções normalizáveis, devemos impor também a condição de que a função de onda seja nula nos extremos, ou seja, $\psi( \pm a)=0$, daí, partindo do conjunto solução 40 obtemos a seguinte equação:

$$
\tan (k a)=-\frac{\hbar^{2} k}{m \alpha}
$$

A equação 41 é uma equação transcendente, onde só é possível determinar soluções numéricas, neste caso, determinar os valores possíveis para $k$ é determinar os valores possíveis para as autoenergias.

\section{Métodos numéricos de soluções}

Em diversos ramos científicos, podemos nos deparar com problemas reais que recaem em situações na qual resolvemos equações na forma $f(x)=0$, onde $f$ é uma função de uma variável real. Resolver a equação $f(x)=0$ consiste em determinar a raiz (ou raízes) real ou complexo $x_{i}$ tal que $f\left(x_{i}\right)=0$. Geometricamente, a raiz real de uma equação é a abscissa do ponto de interseção do gráfico de $f$ com eixo $x$. 
Os valores $\xi_{i}$ determinados são denominados zeros da função $f\left(x_{i}\right)$. Para determinar uma solução real em $f(x)=0$, foram desenvolvidos alguns métodos numéricos que ajudam obter a solução (ou uma aproximação).

Para aplicar tais métodos, é necessário partir de uma aproximação inicial da raiz, e em seguida, fazer um refinamento dessa aproximação por meio de um processo iterativo. Assim, os métodos são constituídos por duas fases:

1. Localizar um intervalo que contenha a raiz.

2. Realizar o refinamento, que consiste em realizar aproximações até obter um raiz aproximada com um precisão $\epsilon$ prefixada.

Para localizar um intervalo que contenha a raiz, é usado frequentemente o teorema de continuidade da funçã ${ }^{3}$ que pode ser visto em [12]. Em determinadas situações, usar somente o teorema esse não é muito apropriado, é fundamental que seja feito uma análise gráfica, permitindo assim, obter uma melhor aproximação da raiz. Assim, utiliza-se programas que traçam gráficos de funções, sendo em algumas calculadores ou softwares matemáticos, por exemplo, GeoGebra 4

\subsection{Método da bisseção}

O método da bisseção 13 consiste, após a localização do intervalo $[a, b]$ que contém a raiz $\xi$, determinar um sequência de intervalos $\left[r_{i}, s_{i}\right]$, sendo $i=0,1,2,3, \ldots$, com $r_{0}=a$ e $s_{0}=b$, de modo que amplitude do intervalo numa iteração é a metade da amplitude do intervalo anterior, sempre contendo a raiz $\xi$. A sequência de intervalos é calculada até que se chegue a um valor menor que um valor $\epsilon$ preestabelecido. Portanto, as sequências são construídas da seguinte forma:

1. Determinar primeiramente um intervalo inicial $\left[r_{0}, s_{0}\right]$ tal que $f\left(r_{0}\right) \cdot f\left(s_{0}\right)<0$.

2. Calcule $x_{i}=\frac{r_{i}+s_{i}}{2}$, ponto médio do intervalo.

3. Se $f\left(x_{i}\right)=0$, então $x_{i}$ é uma raiz de $f$.

4. Se $f\left(r_{i}\right) \cdot f\left(x_{i}\right)<0$, então $r_{i+1}=r_{i}$ e $s_{i+1}=x_{i}$.

5. Se $f\left(r_{i}\right) \cdot f\left(x_{i}\right)>0$, então $r_{i+1}=x_{i}$ e $s_{i+1}=s_{i}$.

A sequência descrita acima pode ser esquematizada segundo a Figura 2 .

O número mínimo $k$ de iterações necessárias para se obter uma boa aproximação da raiz pelo método da bisseção, sendo $\epsilon$ uma precisão preestabelecida, é dada por:

$$
k>\frac{\log \left(s_{0}-r_{0}\right)-\log (\epsilon)}{\log 2}-1,
$$

onde $k$ é o maior inteiro mais próximo.

\footnotetext{
${ }^{3}$ Seja $f$ uma função continua num intervalo $[a, b]$. Se $f(a) \cdot f(b)<0$ então existe pelo menos um ponto $\xi$ entre a e b que é zero de $f$.

${ }^{4}$ GeoGebra é um software gratuito de matemática desenvolvido para o ensino e aprendizagem da matemática, que pode ser baixado no site http://www.geogebra.org/.
}

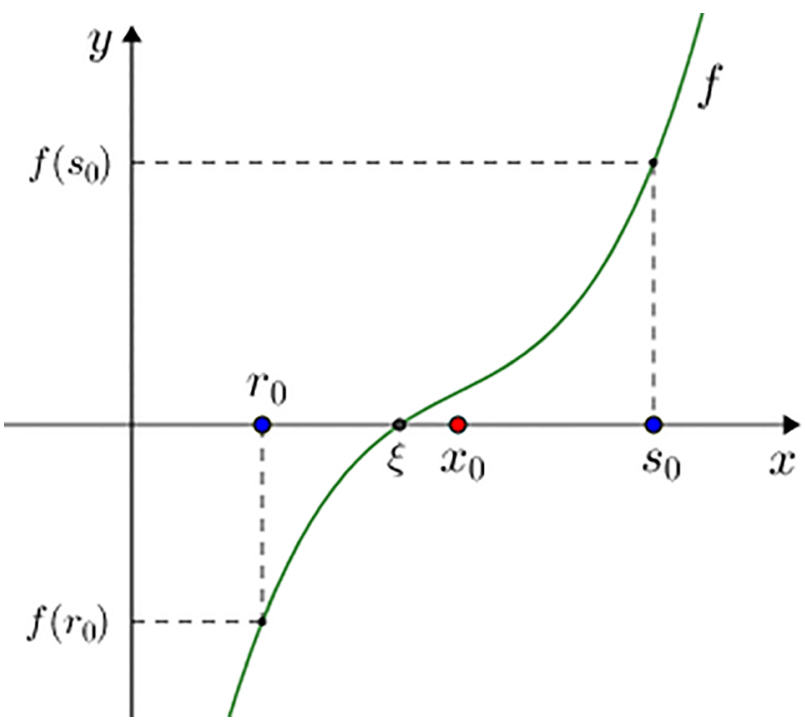

Figura 2: A função arbitrária $f$ é representada pela curva em verde, $r_{0}$ e $s_{0}$ são os pontos que delimitam o intervalo onde a raiz da função se encontra. $\mathrm{O}$ ponto $\xi$ representa a raiz exata enquanto que o ponto $x_{0}$ seria o valor numérico aproximado da raiz.

Convém observar que o método da bisseção gera uma sequência convergente, sendo assim, é sempre possível encontrar um intervalo real que contém a raiz da equação, que satisfaz a precisão previamente definida. As demonstrações das afirmações acima podem ser vistas em 14.

\section{Solução das equações transcendentes}

Retomando ao nosso problema inicial, que consiste em determinar as autoenergias possíveis para os problemas do poço duplo de potencial simétrico tipo $\delta(x)$ e do poço de potencial quadrado infinito com um potencial tipo $\delta(x)$ centrado na origem, representados especificamente pelas equações 30 e (31) para o potencial duplo tipo $\delta(x) \mathrm{e}$ (41) para o problema do poço infinito com potencial $\delta(x)$ centrado.

Esse tipo de problema, comum em mecânica quântica, também pode ser desenvolvido através de rotinas de solução de equações, diferenciais ou não, em programas computacionais voltados para esse fim. No apêndice A. desenvolvemos as soluções numéricas, mostradas a seguir, utilizando o Software Maxima, que consiste num programa computaciona 5

${ }^{5} \mathrm{O}$ Maxima é um sistema de computação algébrica baseado em uma versão de 1982 do Macsyma. Ele é escrito em Common Lisp e funciona em todas as plataformas POSIX, tais como Mac OS $\mathrm{X}$, Unix, BSD, e GNU/Linux bem como no Microsoft Windows. Trata-se de um software livre cuja licença é a GNU General Public License. 


\subsection{Solução das equações 30 e 31 .}

Para resolver estas equações vamos, primeiramente, tomar uma substituição de variáveis da seguinte maneira: tomando $k a=x$, segue que, em (30) e (31), $c x=$ $\hbar^{2} k / m \alpha a$, onde $c=\frac{\hbar^{2}}{m \alpha a}$. Deste modo temos as seguintes equações a resolver:

$$
e^{-2 x}= \pm c x \mp 1
$$

Notemos que o parâmetro $\alpha$ é livre. Assim, vamos tomar $\alpha \sim \hbar^{2} / m a$. Com isto, temos as seguintes equações transcendentes:

$$
e^{-2 x}=x-1
$$

$\mathrm{e}$

$$
e^{-2 x}=-x+1
$$

Da equação (44), com uso do software GeoGebra, plotamos seu comportamento. Como mostrado na Figura 3

Vendo que a solução está entre os valores 1 e 2, definimos que $s_{0}=2$ e $r_{0}=1$. Assim, para uma aproximação de $\epsilon=0.1$ utilizando 42 temos $k=6$, ou seja, para o refinamento de nossa solução vamos repetir o processo da bisseção em 6 vezes.

Tomemos as funções $f$ e $g$, tais que $f(x)=e^{-2 x}$ e $g(x)=x-1$. Definindo a função $h$ tal que $h(x)=$ $f(x)-g(x)$, vamos encontrar o(s) zero(os) da função $h$, o que equivale resolver a equação $e^{-2 x}=x-1$. Assim, temos que:

$$
\begin{aligned}
& x_{0}=\frac{1+2}{2}=1.5 \quad \Longrightarrow\left\{\begin{array}{l}
h(1) \simeq 0.13 \\
h(2) \simeq-0.98 \\
h(1.5) \simeq-0.45
\end{array} \quad \Longrightarrow \quad \xi \in\right] 1,1.5[ \\
& x_{1}=\frac{1+1.5}{2}=1.25 \quad \Longrightarrow\left\{\begin{array}{l}
h(1) \simeq 0.13 \\
h(2) \simeq-0.45 \\
h(1.5) \simeq-0.16
\end{array} \quad \Longrightarrow \quad \xi \in\right] 1,1.25[ \\
& x_{2}=\frac{1+1.25}{2}=1.125 \Longrightarrow\left\{\begin{array}{l}
h(1) \simeq 0.13 \\
h(2) \simeq-0.16 \\
h(1.5) \simeq-0.02
\end{array} \quad \Longrightarrow \quad \xi \in\right] 1,1.125[ \\
& x_{3}=\frac{1+1.125}{2}=1.0625 \Longrightarrow\left\{\begin{array}{l}
h(1) \simeq 0.13 \\
h(2) \simeq-0.02 \\
h(1.5) \simeq 0.05
\end{array} \quad \Longrightarrow \quad \xi \in\right] 1.0625,1.125[ \\
& \text { : } \\
& x_{11}=1.108
\end{aligned}
$$

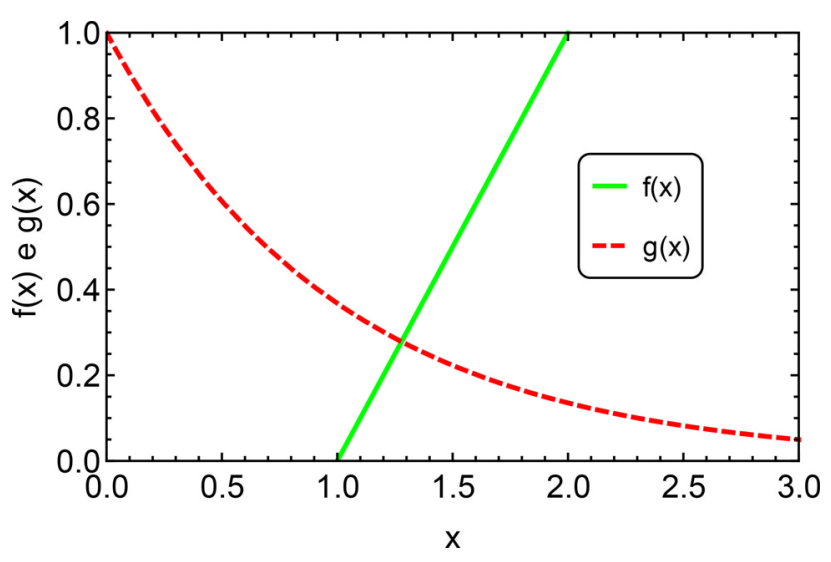

Figura 3: A curva $e^{-2 x}$, em verde, é interceptada pela curva $x-1$, em vermelho, no intervalo entre 1 e 2 .

Determinado o ponto de intersecção entre as curvas em $x=1,108$, definimos que $k a=1,108$ e portanto, utilizando (7) vemos que

$$
E=-1,227 \frac{\hbar^{2}}{2 m a^{2}}
$$

define a energia do único estado ligadd ${ }^{6}$

Vamos resolver agora a equação 45 de maneira análoga ao que foi desenvolvido para a equação (44). Para observar o comportamento de (45) vamos, primeiramente, plotar seu gráfico, como é mostrado na Figura 4

De fato, analisando o gráfico definido pela Figura 4 e tomando a equação (42), é fácil ver que $k=6$ é o número mínimo de interações para se obter um resultado com boa aproximação numérica. Seguindo os mesmos passos para determinarmos a solução numérica de 44 encontramos para (45) que $x=0.799 \mathrm{com}$ precisão de três cassas decimais e, portanto, $k a=0.799$. utilizando

${ }^{6} \mathrm{O}$ desenvolvimento numérico utilizado para chegarmos na altoenergia definida para o único estado ligado, equação 46 foi desenvolvido seguindo a rotina de programação definida em A.2 enquanto que, para a equação 47 seguimos a rotina de programação definida em A.3. 


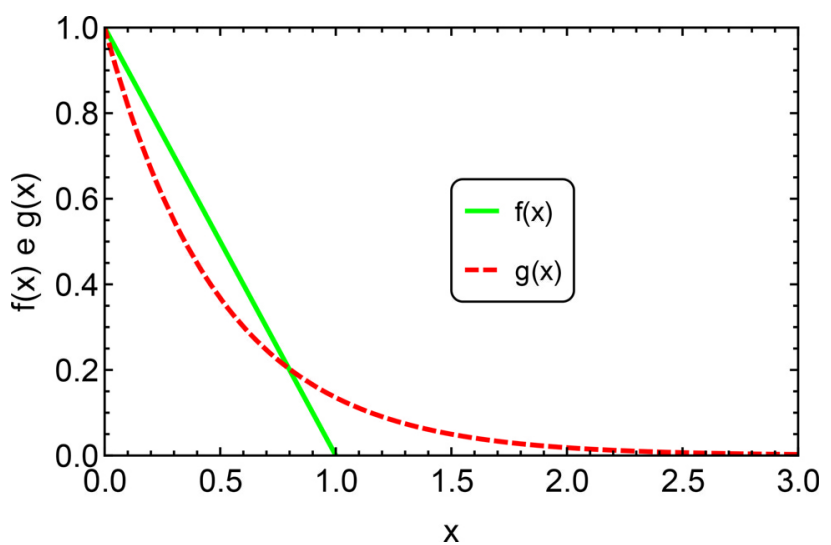

Figura 4: A curva $e^{-2 x}$, em verde, é interceptada pela curva $1-x$, em vermelho em dois pontos, um em zero, e outro entre zero e 1 .

a equação (7) temos

$$
E_{1}=-0.638 \frac{\hbar^{2}}{2 m a^{2}}
$$

\subsection{Solução da equação 41}

Tomando a substituição $k a=x$ em 41) obtemos a seguinte equação transcendente:

$$
\tan x=-c x
$$

onde $c=\frac{\hbar^{2}}{m \alpha a}$.

Chamando $f(x)=\tan x$ e $g(x)=-c x$, definindo a função $h$ dada por $h(x)=f(x)-g(x)$ vamos determinar os zeros da função $h$ e por consequência, determinar a solução de 41. Para isso, plotando o gráfico de 48, como mostrado na Figura 5 , escolhendo o parâmetro livre tal que $\alpha \sim 2 \hbar^{2} / m a$, devemos resolver numericament $7^{7}$ a equação:

$$
\tan x=-\frac{x}{2}
$$

Por exemplo, utilizando o método da bisseção pode-se mostrar que numericamente, para o primeiro estado acessível tem-se a solução numérica tan $x=x / 2=2.288$, com precisão de três casas decimais, que é um valor numérico maior que $\pi / 2$ e menor que $\pi$. Por consequência, repetindo este procedimento para as autoenergias acessíveis subsequentes, é possível ver que estas também estarão deslocadas acima dos valores de $n$ inteiros e ímpar, isto é, as autoenergias para o poço quadrado infinito com um potencial tipo $\delta$ de Dirac centrado na origem são aquelas em que:

$$
E_{n} \gtrsim \frac{n^{2} \pi^{2} \hbar^{2}}{2 m(2 a)^{2}} \forall n=1,3,5 \ldots
$$

A respeito das solução de autoenergia para este caso, podemos também levantar alguns comportamentos analiticamente. Notemos que a escolha do parâmetro livre

\footnotetext{
${ }^{7}$ Numericamente, escolhemos o método da bisseção para resolução dessas equações, definido na rotina de programação em A.4
}

$\alpha$ influencia diretamente nas autoenergias possíveis. A medida que $\alpha \rightarrow 0$ notemos, pela Figura 5 , que a função $g(x)$ se torna mais e mais inclinada, desta maneira as soluções possíveis irão se aproximar das soluções do poço quadrado infinito sem o potencial $\delta$ de Dirac centrado na origem. Por outro lado, se fizermos $\alpha \rightarrow \infty$ notamos que a função $g(x)$ se aproxima mais e mais do eixo das abcissas e as intersecções que delimitam os estados de energia possíveis irão ocorrer justamente nos pontos $n \pi(\forall n=1,3,5, \ldots)$. Deste modo, as autoenergias possíveis se aproximam das autoenergias do poço quadrado infinito de largura $a$, ou seja:

$$
E_{n} \rightarrow \frac{n^{2} \pi^{2} \hbar^{2}}{2 m a^{2}}
$$

Neste limite, a barreira de potencial tipo $\delta(x)$ se torna impenetrável e o problema em questão passaria a ser tratado como dois poços de potenciais quadrado infinitos isolados um do outro.

\section{Conclusões}

O problema proposto por este trabalho foi trabalhar aspectos do cálculo numérico dentro da resolução de problemas envolvendo a mecânica quântica especificamente em dois casos: $(i) \mathrm{O}$ potencial duplo tipo $\delta(x)$. E o (ii) O poço de potencial quadrado infinito com uma descontinuidade, $\delta(x)$, na origem.

Para tratar dos problemas propostos, encontramos, primeiramente, as equações transcendentes dadas pelas equações (30) e (31) para o caso $(i)$, e pela equação (41) para o caso $(i i)$. Nos dois casos é visto que a paridade da função de onda, que satisfaz a equação de Schrödinger independente do tempo, influencia diretamente nos estados fisicamente acessíveis.

Diante das equações (30), 31) e 41, fizemos uma revisão sobre cálculo numérico e apresentamos o método de resolução de equações pela bisseção, que consiste num

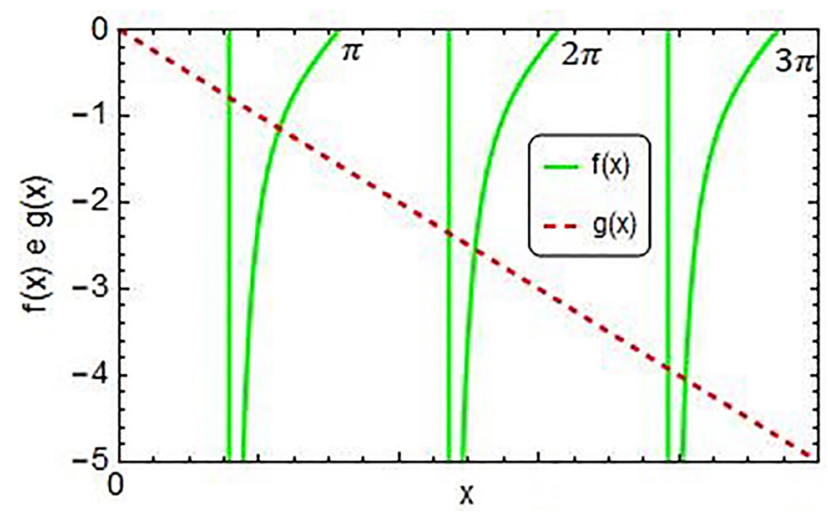

Figura 5: A função $f(x)=\tan x$, é representada pela curva em verde, enquanto que a função $g(x)=-x / 2$ é representada pela reta tracejada em vermelho. O parâmetro livre $\alpha$ foi escolhido tal que $\alpha \sim 2 \hbar^{2} / m a$. Os pontos de interseção das duas funções representam as soluções possíveis. 
método aproximativo para determinação as raízes de equações.

Por fim, determinamos, pelo método da bisseção, as autoenergias possíveis para o caso $(i)$ de tal modo que, para soluções de paridade positiva existe apenas um autoestado cuja a autoenergia é definida pela equação 46 . Por outro lado, para as soluções com paridade ímpar mostra-se graficamente, a partir da Figura 4, que há no máximo dois autoestados possíveis e que a energia do maior autoestado é dada pela equação 47). Nos dois casos como o parâmetro $\alpha$ é um parâmetro livre as autoenergias determinadas foram obtidas para a escolha $\alpha \sim \hbar^{2} / m a$.

Para o caso (ii) a equação transcendente a se resolver foi determinada pela equação (48). A partir dela mostrou-se que as autoenergias permitidas para este caso devem ser ligeiramente maiores para que as autoenergias permitidas para o poço quadrado de potencial infinito simples de largura $2 a$ quando tratamos das soluções pares da equação de Schrödinger independente do tempo. Alem disso, mostramos que dependendo da escolha do parâmetro livre $\alpha$ as autoenergias tendem para as autoenergias do poço quadrado de potencial infinito de largura $2 a$ (para $\alpha \rightarrow 0)$. Ou para um problema de dois poços quadrados infinitos simétricos de largura $a$ separados por uma barreira intransponível centrada na origem (para $\alpha \rightarrow \infty)$.

\section{Agradecimentos}

Gostaríamos de agradecer à UFRR pela infra-estrutura de trabalho, em especial ao Colégio de Aplicação da UFRR pela organização e disponibilidade de recursos para o desenvolvimento deste trabalho.

\section{A. Material suplementar}

\section{A.1. Rotina de programação no software MAXIMA}

O MAXIMA, permite manipular e explorar expressões matemáticas de maneira simbólica e interativa. O usuário digitaliza na janela do programa algumas fórmulas, comandos e o sistema avalia-os devolvendo uma resposta que pode ser manipulada posteriormente, caso seja necessário, permitido também obter soluções numéricas aproximadas e visualizar graficamente. O MAXIMA, como se trata de um software do tipo "freeware", com funcionalidades similares aos softwares comercializados, não estimula o uso de cópias não autorizadas. A seguir mostraremos a rotina desenvolvida no software $M A X I M A$ para executar os cálculos numéricos

\section{A.2. Rotina de programação para o cálculo da equação (44)}

Inicialmente, definimos o gráfico da função definida pela Eq. (44), conforme é feito em na saída $(\% \circ 1)^{8}$

$\longrightarrow \operatorname{plot2d}\left(\exp \left(-2^{*} \mathrm{x}\right)-\mathrm{x}+1,[\mathrm{x}, 0,4]\right.$, $[\mathrm{y},-5,5],[\mathrm{ylabel}, " \mathrm{f}(\mathrm{x}) "])$;

C:/Users/Mikael/AppData/Local/Temp

$$
\text { /maxout12392.gnuplot? }
$$

A seguir, em (\%i3), definimos os valores numéricos do intervalo em que a raíz da função se encontra. Note que aentrada (\%i4), vai calcular exatamente os valores de $\$ \mathrm{f}(\mathrm{x}) \$$ no intervalo definido.

$\left(\begin{array}{l}\% \\ \text { i3 }\end{array}\right) \quad \mathrm{f}(\mathrm{x}):=$ float $\left(\exp \left(-2^{*} \mathrm{x}\right)-\mathrm{x}+1\right) \$$

$$
[\mathrm{x} 1, \mathrm{x} 2]:[1 ., 2] \$
$$

(\% i4) $\quad[\mathrm{f}(\mathrm{x} 1), \mathrm{f}(\mathrm{x} 2)]$;

$$
\text { [0.1353352832366127, -0.9816843611112658] }
$$

Por fim, a entrada (\%i6) irá calcular o número de vezes em que a rotina irá trabalhar, onde o termor\$abs $(\mathrm{x} 2$ x1) $>0.00001 \$$ é a precisão.

(\% i6)

$[\mathrm{x} 1, \mathrm{x} 2]:[1 ., 2] \$$

while abs $(\mathrm{x} 2-\mathrm{x} 1)>0.00001$

$\operatorname{do}(x m:(x 1+x 2) / 2$, if $f(x 1) * f(x m)>0$ then $x 1: x m$ else $\mathrm{x} 2: \mathrm{xm}$,

$\operatorname{print}(\mathrm{xm}))$, numer $\$$

$$
\begin{gathered}
1.5 \\
1.25 \\
1.125 \\
1.0625 \\
1.09375 \\
1.109375 \\
1.1015625 \\
1.10546875 \\
1.107421875 \\
1.1083984375 \\
1.10888671875
\end{gathered}
$$

A raiz, com precisão de 3 casas decimais é 1.108 . Observe-se que a função já tinha sido definida previamente e que já estava garantido que os sinais da função são diferentes nos dois pontos iniciais.

8 As saídas (\%01), (\%07) e (\%04) são, respectivamente, os gráficos dados pelas Figuras 3 e 5

${ }^{9}$ Este caminho apresentado indica o local, no computador que está rodando o MAXIMA, onde será armazenada o gráfico gerado para análise numérica da função. 


\section{A.3. Rotina de programação para o cálculo da equação 45}

Para a equação 45, temos:

(\% i7)

$\operatorname{plot} 2 \mathrm{~d}\left(\exp \left(-2^{*} \mathrm{x}\right)+\mathrm{x}-1,[\mathrm{x}, 0,1]\right.$, $[\mathrm{y},-0.5,0.5],[$ ylabel, $" \mathrm{~g}(\mathrm{x}) "])$;

$$
\text { C:/Users/Mikael/AppData/Local/ （\% o7) }
$$

Temp/maxout12392.gnuplot

(\% i9) $\mathrm{g}(\mathrm{x}):=$ bfloat $\left(\exp \left(-2^{*} \mathrm{x}\right)-\mathrm{x}+1\right) \$$ $[\mathrm{x} 1, \mathrm{x} 2]:[0.5,0.8] \$$

(\% i10) $[\mathrm{g}(\mathrm{x} 1), \mathrm{g}(\mathrm{x} 2)]$;

$$
[8.67879441 b-1,4.018965179 b-1] \quad(\% \text { o } 10)
$$

\section{(\% i12)}

$[\mathrm{x} 1, \mathrm{x} 2]:[0.5,0.8] \$$

while $\operatorname{abs}(\mathrm{x} 2-\mathrm{x} 1)>0.0001$

$\operatorname{do}(\mathrm{xm}:(\mathrm{x} 1+\mathrm{x} 2) / 2$, if $\mathrm{g}(\mathrm{x} 1) * \mathrm{~g}(\mathrm{xm})>0$

then $\mathrm{x} 1: \mathrm{xm}$ else $\mathrm{x} 2: \mathrm{xm}$,

print $(\mathrm{xm}))$,numer\$

$$
\begin{gathered}
0.65 \\
0.725 \\
0.7625 \\
0.78125 \\
0.790625 \\
0.7953125 \\
0.79765625 \\
0.798828125 \\
0.7994140625 \\
0.79970703125
\end{gathered}
$$

\section{A.4. Rotina de programação para o cálculo da equação 48}

Para a equação 48 temos:

(\% i17) $\operatorname{plot} 2 \mathrm{~d}(\tan (\mathrm{x})+\mathrm{x} / 2,[\mathrm{x}, 0.001, \% \mathrm{pi}]$, $[\mathrm{y},-3,0.001],[$ ylabel,, $\mathrm{h}(\mathrm{x}) "])$;

plot2d: some values were clipped.

$$
\text { C:/Users/Mikael/AppData/Local/ }
$$

$$
\text { Temp/maxout12392.gnuplot }
$$

(\% i19) $\mathrm{h}(\mathrm{x}):=$ bfloat $(\tan (\mathrm{x})+\mathrm{x} / 2) \$$

(\% i20) $[\mathrm{h}(\mathrm{x} 1), \mathrm{h}(\mathrm{x} 2)]$;

$$
[-1.185039 b 0,5.029777 b-1]
$$

(\% i22) $[\mathrm{x} 1, \mathrm{x} 2]:[2,2.5] \$$

while $\operatorname{abs}(\mathrm{x} 2-\mathrm{x} 1)>0.0001$

$\operatorname{do}(\mathrm{xm}:(\mathrm{x} 1+\mathrm{x} 2) / 2$, if $\mathrm{h}(\mathrm{x} 1) * \mathrm{~h}(\mathrm{xm})>0$

then $\mathrm{x} 1: \mathrm{xm}$ else $\mathrm{x} 2: \mathrm{xm}$, $\operatorname{print}(\mathrm{xm}))$,numer\$

$$
\begin{gathered}
2.25 \\
2.375 \\
2.3125 \\
2.28125 \\
2.296875 \\
2.2890625 \\
2.28515625 \\
2.287109375 \\
2.2880859375 \\
2.28857421875
\end{gathered}
$$

\section{Referências}

[1] S. Gasiorowicz, Física Quântica (Guanabara Dois, Rio de Janeiro, 1974).

[2] C. Cohen-Tannoudji, B. Diu e F. Laloë, Quantum Mechanics (Hermann, Paris, 1977).

[3] A. Galindo e R. Pascual, Quantum Mechanics I (Springer-Verlag, Berlim, 1990).

[4] K. Gottfried e T.M. Yan, Quantum Mechanics: Fundamentals (Springer, Nova Iorque, 2003).

[5] K. Tamvakis, Problems and Solutions in Quantum Mechanics (Cambridge University Press, Cambridge, 2005).

[6] R.W. Robinett, Quantum Mechanics (Oxford University Press, Oxford, 2006), $2^{\mathrm{a}}$ ed.

[7] D.J. Griffiths, Mecânica Quântica (Pearson Prentice Hall, São Paulo, 2011), $2^{\mathrm{a}}$ ed.

[8] A.S. de Castro, Revista Brasileira de Ensino de Física 34, 4301 (2012).

[9] A.S. de Castro, Revista Brasileira de Ensino de Física 34, 4304 (2012).

[10] T.V. Cardoso, Aplicação dos métodos Númerov e Simpson na resolução de auto-consistente da equação de Schrodinger. Trabalho de Conclusão de Curso, Universidade Federal de Uberlândia, Uberlândia (2010).

[11] http://www.decom.ufop.br/marcone/Disciplinas/ CalculoNumerico/Equacoes.pdf

[12] M.A. Munem e D. Foulis, Cálculo (Guanabara Dois, Rio de Janeiro, 1982), v. 1.

[13] M.A.G. Ruggiero e V.L.R. Lopes, Cálculo Numérico, Aspectos Teóricos e Computacionais (McGraw-Hill, São Paulo, 1988).

[14] S. Arenales e A. Darezzo, Cálculo numérico: aprendizagem com apoio de software (Thomson Learning, São Paulo, 2008). 